\title{
Electrospun fixed dose formulations of amlodipine besylate and valsartan
}

\author{
Haitham Bukhary, Gareth R. Williams, ${ }^{*}$ and Mine Orlu* \\ UCL School of Pharmacy, University College London, 29-39 Brunswick Square, London, WC1N 1AX, UK \\ * authors for correspondence. Email: g.williams@ucl.ac.uk (GRW); m.orlu@ucl.ac.uk (MO).Tel: +44 (0) \\ 2077535868 (GRW); +44 (0) 2077535968 (MO).
}

\begin{abstract}
Increasing numbers of elderly people require multi-drug therapies. One route to improve adherence rates is to prepare fixed dose combinations (FDCs), in which multiple active ingredients are loaded into a single formulation. Here, we report the use of electrospinning to prepare fast-dissolving oral FDCs containing amlodipine besylate and valsartan, two drugs prescribed as FDCs for the treatment of hypertension. Electrospun fibers were prepared loaded with one or both drugs, using polyvinylpyrrolidone as the polymer matrix. The fibers were cylindrical in morphology and comprise amorphous solid dispersions except with the highest loadings of amlodipine besylate. HPLC demonstrated drug entrapment efficiencies of between 90 and $99 \%$ of the theoretical dose. The mats have folding endurances and thicknesses suitable for use as oral films. The amlodipine besylate-loaded systems are fast-dissolving, with $100 \%$ release obtained within $120 \mathrm{~s}$. In contrast, valsartan release from its single-drug formulations took longer, ranging from $360 \mathrm{~s}$ to $24 \mathrm{~min}$. With the FDC formulations, rapid release within $360 \mathrm{~s}$ was achieved when the loading was $5 \% \mathrm{w} / \mathrm{w}$ of each drug, but again the release time increased with drug loading. Electrospun fibers therefore have significant promise as FDCs, but the target drug and its loading need to be carefully considered.
\end{abstract}

Keywords: Fixed dose combinations, electrospinning, polyvinylpyrrolidone, amlodipine besylate, valsartan, fast dissolving films.

\section{Introduction}

In the UK, older people make up $20 \%$ of the population and consume almost $50 \%$ of prescription drugs (Gorard, 2006). The majority of people aged 65 and older are diagnosed with multiple diseases that require management with several active ingredients simultaneously, often to the extent of polypharmacy, which is loosely defined as synchronous use of two to five or more medicines (Fulton and Allen, 2005). The adherence of patients to their drug regimen is crucial for successful therapeutic outcomes (Jimmy and Jose, 2011), and patients involved in polypharmacy are likely to find it challenging to take all their medicines at the appropriate times. This can profoundly affect the patient's quality of life, and also adds extra economic costs for healthcare providers (Hughes, 2004). Therefore, it is the task of formulators to prepare medicines to minimize the burden on patients and maximize the likelihood of a dosage regimen being accurately followed. 
Much work has been devoted to ensuring formulations have good patient acceptability (Liu et al., 2014), for instance to ensure ease of swallowing: this is key in designing geriatric oral formulations, in order to prevent oesophageal retention and risk of aspiration (Liu et al., 2014). An attractive solution to overcome the acceptability challenge is simplifying the treatment regimen for multiple drugs, by combining them in a single dosage form. Fixed dose combination (FDC) drug products are defined as those which combine two or more active pharmaceutical ingredients (APIs) in a single dosage form at a fixed dose ratio. FDCs are designed to facilitate simpler treatment plans for multiple drugs which have similarly timed regimens (Bangalore et al., 2007), and thereby improve patient compliance (European Medicines Agency, 2017). FDCs are expected to have particular promise for patients suffering from chronic conditions like hypertension and diabetes, where long term medication is required (Desai et al., 2013). It has been suggested that a $26 \%$ reduction in older patient non-adherence should be achievable using FDCs (Bangalore et al., 2007). However, FDC tablets or capsules tend to be larger than the individual drug formulations, and thus if poorly designed FDCs could in fact reduce patient compliance because of swallowing difficulties (European Medicines Agency, 2017).

A number of conventional pharmaceutical technologies have been used for commercial FDC product manufacturing (Desai et al., 2013), such as the incorporation of multiple drugs in separate layers of multilayer tablets (Mitra and $\mathrm{Wu}, 2012$ ). Emerging technologies have also been investigated: for instance, 3D printing has been employed to produce a polypill containing five different APIs (Khaled et al., 2015). An alternative which has to date not been widely explored in the context of FDCs is electrospinning. This is a simple technique in which electrical energy is applied to a polymer/drug solution, usually resulting in amorphous solid dispersions (ASDs) in the form of nanoscale fibers. The production of ASDs can lead to enhanced dissolution and solubility, attractive for preparing formulations of APIs with low water solubility (Williams et al., 2012; Zamani et al., 2013). A range of polymers can be processed, and multiple active pharmaceutical ingredients incorporated into a single fiber formulation (Illangakoon et al., 2014; Li et al., 2013a).

As a result, electrospinning has been widely explored as a method for fabricating fast dissolving thin films (Williams et al., 2012). For example, an oral fast-dissolving drug delivery system was prepared from the hydrophilic polymer polyvinylpyrrolidone (PVP) and ibuprofen, and complete release of the drug observed in $10 \mathrm{~s}$ (Yu et al., 2009). Other fast-dissolving drug-loaded PVP fibres have been reported containing a wide variety of active ingredients, including ketoprofen (a non-steroidal anti-inflammatory drug) (Yu et al., 2010), irbesartan (used to treat high blood pressure) (Adeli, 2015), vitamin D (Li et al., 2013b), or isosorbide dinitrate (used in the treatment of angina),(Chen et al., 2016). Very rapid release can be achieved with multiple APIs in the same fiber formulation, as has been seen for electrospun polyvinylalcohol fibers loaded with caffeine and riboflavin (Li et al., 2013a) and PVP fibers containing paracetamol and caffeine (Illangakoon et al., 2014).

Fast-dissolving oral drug delivery systems have the advantage of immediate disintegration and drug dissolution occurring in the mouth, providing rapid onset of action (Seager, 1998). Since the formulation does not need to be taken with water, they can be used for patients suffering from dysphagia, common in the elderly. Sufficiently high aqueous solubility is crucial for their success, however. This requirement 
104

105

106

107

108

109

110

111

112

becomes increasingly problematic with the increasing number of poorly water soluble drugs emerging onto the market (Nagy et al., 2010).

Amlodipine besylate and valsartan are frequently prescribed in combination as blood pressure lowering agents for the treatment of hypertension when monotherapy is not sufficient (Plosker and Robinson, 2008). An FDC (EXFORGE ${ }^{\circledR}$ ) is available in the form of film coated tablets containing amlodipine besylate and valsartan in doses of 5/80 mg, 5/160 mg, and 10/160 mg. Both drugs have low aqueous solubility, and hence a fast-dissolving oral formulation would be highly valuable.

The aim of this project was to investigate the use of electrospinning to prepare FDCs of valsartan and amlodipine besylate, with the intended application as oral fast dissolving films. A series of single-drug and dual-drug loaded systems were prepared and fully characterized, and their disintegration and drug release evaluated.

\section{Experimental details}

2.1 Materials

Polyvinylpyrrolidone (PVP; MW 360,000 Da) and amlodipine besylate (AB) were purchased from SigmaAldrich, UK. Valsartan (VAL) was sourced from LKT Laboratories Inc., USA. Analytical grade ethanol, acetonitrile and methanol were procured from Sigma-Aldrich, UK. Deionised water was used for all studies, and all other chemicals used were of analytical grade.

\subsection{Preparation of spinning solutions}

$10 \% \mathrm{w} / \mathrm{v}$ PVP solutions were prepared in ethanol, with stirring overnight to ensure complete dissolution. Drug-loaded solutions were prepared as detailed in Table 1, by pre-dissolving the required amount of $A B$ or/and VAL in $2 \mathrm{~mL}$ of ethanol, then combining this with $5 \mathrm{~mL}$ of the PVP solution. Mechanical stirring was applied at room temperature for $20 \mathrm{~min}$ until homogeneous solutions were formed. Drug/polymer physical mixtures (PM) were prepared for control purposes.

Table 1. The compositions of the spinning solutions.

\begin{tabular}{|c|c|c|c|c|}
\hline & \multirow{2}{*}{ ID } & \multirow{2}{*}{ Drug loading $(\% \mathrm{w} / \mathrm{w})$} & \multicolumn{2}{|c|}{ Drug mass in $2 \mathrm{~mL}$ ethanol $(\mathrm{mg})$} \\
\hline & & & $A B$ & VAL \\
\hline \multirow{4}{*}{$A B$ formulations } & $\mathrm{A} 1$ & 5 & 26 & - \\
\hline & $\mathrm{A} 2$ & 15 & 88 & - \\
\hline & A3 & 30 & 214 & - \\
\hline & A4 & 55 & 611 & - \\
\hline \multirow{4}{*}{ VAL formulations } & V1 & 5 & - & 26 \\
\hline & V2 & 15 & - & 88 \\
\hline & V3 & 30 & - & 214 \\
\hline & V4 & 55 & - & 611 \\
\hline \multirow{3}{*}{ FDCs } & AV1 & $5 / 5 \mathrm{AB} / \mathrm{VAL}$ & 28.5 & 28.5 \\
\hline & AV2 & 15 / 15 AB / VAL & 107 & 107 \\
\hline & AV3 & 30 / $30 \mathrm{AB} / \mathrm{VAL}$ & 375 & 375 \\
\hline
\end{tabular}




\subsection{Electrospinning}

Polymer solutions were loaded into $5 \mathrm{~mL}$ plastic syringes (Terumo, MediSupplies, UK) fitted with stainless steel dispensing needles (20G, $0.61 \mathrm{~mm}$ inner diameter, Nordson EFD, UK) with care taken to avoid any air bubbles. A voltage of $+15 \mathrm{kV}$ was applied to the needle (spinneret) using a HCP 35-35000 power supply (FuG Elektronik GmbH, Germany). The feed rate of the solution was controlled with a syringe pump (KDS100, Cole Parmer, UK) at $1.3 \mathrm{~mL} \mathrm{~h}^{-1}$, and $5 \mathrm{~mL}$ of fluid was dispensed. The fibers were collected on a flat grounded collector $(20 \times 30 \mathrm{~cm})$ covered with aluminum foil and situated $12 \mathrm{~cm}$ from the spinneret tip. Electrospinning was carried out under ambient conditions $\left(18-23{ }^{\circ} \mathrm{C}\right.$ and relative humidity of $26-63 \%)$. After fabrication, the electrospun fiber mats were stored in a vacuum desiccator over silica gel beads to aid evaporation of the remaining organic solvents and moisture.

\subsection{Characterization \\ 2.4.1 Scanning electron microscopy}

The fiber morphology was assessed by scanning electron microscopy (SEM; Quanta 200 FEG ESEM, FEI, USA). Prior to examination, samples were sputter-coated with gold under argon to make them electrically conductive. Images were taken at an excitation voltage of $5 \mathrm{kV}$. The average diameter of the fibers was determined from the SEM images by manual measurements at 100 different points using the ImageJ software (National Institutes of Health, USA).

\subsubsection{Thickness of the fiber mat}

$5 \mathrm{~mL}$ of each solution was electrospun onto aluminium foil. A $4 \mathrm{~cm}$ diameter circular section was cut from three different locations on the foil with a biopsy punch, and the thickness of each measured using electronic digital Vernier callipers. Data are presented as mean \pm S.D. $(n=3)$.

\subsubsection{Folding endurance}

The brittleness of the fiber mats was quantified in terms of the folding endurance, which is defined as the number of times the mat can be folded at the same place without breaking or cracking (Mundargi et al., 2007). A circular section of $4 \mathrm{~cm}$ diameter cut with the biopsy punch was placed in test apparatus built in-house. This is designed to sequentially fold the fiber mat around the central diameter (see Supporting Information, Figure S1). Each fold rotates $180^{\circ}$ around the central radius $\left(+90^{\circ}\right.$ and $\left.-90^{\circ}\right)$. The number of times the mat could be folded until cracks appeared on the fold line was counted (Figure S1). Data from each formulation sample are presented as mean \pm S.D. $(n=3)$.

\subsubsection{X-ray diffraction}

X-ray diffraction patterns were obtained on a MiniFlex 600 Diffractometer (Rigaku, Japan) supplied with $\mathrm{Cu} \mathrm{K} \alpha$ radiation $(\lambda=1.5418 \AA$ ) at a voltage of $40 \mathrm{kV}$ and current of $15 \mathrm{~mA}$. Samples were fixed on an aluminium holder and data recorded over the $2 \theta$ range between 3 to $45^{\circ}$ at a scan speed of $5^{\circ} \mathrm{min}^{-1}$.

\subsubsection{Differential scanning calorimetry}

Differential scanning calorimetry (DSC) studies were conducted using a Q2000 calorimeter (TA instruments, USA). Non-hermetically sealed samples in aluminium pans (Tzero premium pan/lid, TA instruments) were heated over the range 30 to $180{ }^{\circ} \mathrm{C}$ for VAL-based materials, or 30 to $220{ }^{\circ} \mathrm{C}$ for 
formulations containing $\mathrm{AB}$. Experiments were undertaken at a rate of $10^{\circ} \mathrm{C} \mathrm{min}^{-1}$ and under a nitrogen flow of $50 \mathrm{~mL} \mathrm{~min}^{-1}$. Data were analysed using the TA instruments Universal Analysis software.

\subsubsection{Fourier transform infrared spectroscopy}

Infrared (IR) spectra were collected with the aid of a Spectrum 100 spectrometer (PerkinElmer, USA). Samples were studied over the range 4000 to $650 \mathrm{~cm}^{-1}$, with the spectral resolution set at $1 \mathrm{~cm}^{-1} .4$ scans per sample were recorded.

\subsubsection{Drug loading and entrapment efficiency}

A high performance liquid chromatography (HPLC) method was developed to simultaneously detect both $A B$ and VAL. This is similar to that previously reported by (Celebier et al., 2010). Samples of $5 \pm 0.5$ $\mathrm{mg}$ were dissolved in $50 \mathrm{~mL}$ of methanol then diluted to a final volume of $100 \mathrm{~mL}$ with deionised. The resultant solution was sonicated in a water bath for $30 \mathrm{~min}$ to ensure complete dissolution.

A calibration curve for $A B$ and VAL was prepared from fresh solutions on the day of the experiment. $A$ quaternary HPLC pump (1200 Infinity Series, Agilent technologies, USA) was used to mix the mobile phase, which comprised 30:35:35 v/v/v phosphate buffered saline (PBS; $\mathrm{pH}$ 3.6, $0.01 \mathrm{~mol} \mathrm{~L}^{-1}$ ) : acetonitrile : methanol. Adjustment of the PBS pH was undertaken using orthophosphoric acid (85\%, HPLC grade, Fisher Scientific, UK) and the solution filtered through a $0.45 \mu \mathrm{m}$ membrane filters (Millex syringe filter, Merck Millipore, Germany) before use. The stationary phase used was a C18(2) column (00G-4252-E0, Phenomenex Luna, UK). The injected volume was $20 \mu \mathrm{L}$, the mobile phase flow rate $1 \mathrm{~mL}$ $\mathrm{min}^{-1}$, and the column temperature $40^{\circ} \mathrm{C}$. A UV detector was used for quantification at $240 \mathrm{~nm}$, where $A B$ can be detected at $t=4.9$ min and $V A L$ at $t=7.2 \mathrm{~min}$.

The drug loading and entrapment efficiency (\%) were calculated from equations (1) and (2). Values are recorded as the mean \pm S.D. of 3 independent experiments using a single batch of fibers.

Drug loading $(\%)=[$ mass of drug in fiber mat $\div$ mass of fiber mat $] \times 100$

Entrapment efficiency (\%) $=$ [mass of drug in fibers $\div$ mass of drug in feed solution $] \times 100$

\subsubsection{Wetting assays}

A $4 \mathrm{~cm}$ diameter circular section of the fiber mat was dropped into an $8 \mathrm{~cm}$ Petri dish containing $15 \mathrm{~mL}$ of simulated saliva (SS) solution under stirring at $150 \mathrm{rpm}$. The latter was prepared by mixing $8 \mathrm{~g} \mathrm{NaCl}$, $0.19 \mathrm{~g} \mathrm{KH}_{2} \mathrm{PO}_{4}$, and $2.38 \mathrm{~g} \mathrm{Na}_{2} \mathrm{HPO}_{4}$ in $1 \mathrm{~L}$ of distilled water. Experiments were performed at room temperature. The wetting and disintegration of the fiber mats were recorded with a high-speed camera (HotShot 1280 CC, NAC Image Technology, Japan) at 500 frames per second.

\subsubsection{In vitro drug release studies}

The intended route of fiber administration is as a fast dissolving oral film. Standard USP methods for dissolution testing do not accurately mimic this, and hence a more realistic in vitro release study was employed, similar that reported previously (Illangakoon et al., 2014). $15 \mathrm{~mL}$ SS was first pre-warmed to 
$37.5 \pm 2{ }^{\circ} \mathrm{C}$, and transferred into a $8 \mathrm{~cm}$ Petri dish held in an oil bath at $37^{\circ} \mathrm{C}$. A sample of the desired

200

201

202

203

204

205

206

207

208

209

210

211

212

213 formulation $(5 \pm 0.5 \mathrm{mg}$ ) was dropped into the warm SS, and the mixture stirred with a $1 \mathrm{~cm}$ magnetic follower at $150 \mathrm{rpm}$. At predetermined time points a $200 \mu \mathrm{L}$ sample was taken from the Petri dish for HPLC analysis and replaced with an equal volume of fresh pre-warmed SS, to maintain a total volume of $15 \mathrm{~mL}$. Three independent experiments were carried out and cumulative release percentages are reported as mean \pm S.D.

\subsubsection{Stability study}

The physical form of the components in the electrospun nanofibers was assessed after storage over silica gel in a desiccator for 4 months, using XRD and DSC as detailed above.

\section{Results and discussion}

\subsection{Fiber morphology}

SEM images of the nanofibers from all formulations are presented in Figure 1.
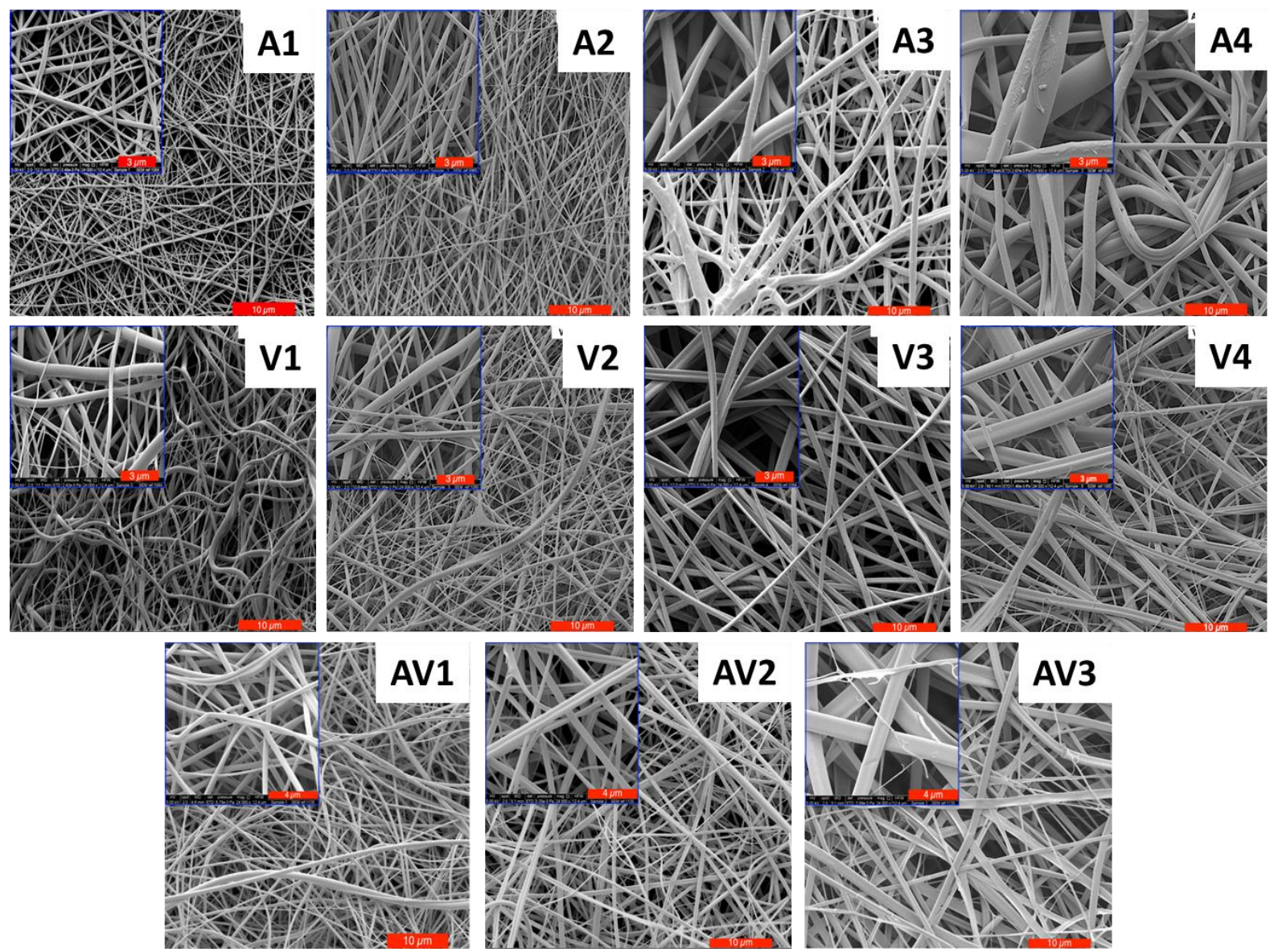

Figure 1: SEM images of the fiber formulations, showing A1 (5\% w/w AB); A2 (15\% w/w AB); A3 (30\% w/w AB); A4 (55\% $\mathrm{w} / \mathrm{w} \mathrm{AB}) ; \mathrm{V} 1$ ( $5 \% \mathrm{w} / \mathrm{w}$ VAL); V2 (15\% w/w VAL); V3 (30\% w/w VAL); V4 ( $55 \% \mathrm{w} / \mathrm{w}$ VAL); AV1 (5\% w/w AB, $5 \% \mathrm{w} / \mathrm{w}$ VAL); AV2 (10\% w/w AB, 10\% w/w VAL); and, AV3 (30\% w/w AB, 30\% w/w VAL). Scale bars: main images $10 \mu \mathrm{m}$; insets for $\mathrm{A} 1$ $A 4$ and $V 1-V 43 \mu \mathrm{m}$; insets for AV1 - AV3 $4 \mu \mathrm{m}$. 
The images show that fibers have formed in all cases. The AB-loaded fibers A1 to A4 all have smooth cylindrical morphologies, with some small particles visible in A4. In contrast, the VAL-containing fibers V1 to V4 and AV1 to AV3 change from cylindrical shapes to flattened ribbon-like fibers as the drug loading increases. This may be a result of the solution having high viscosity at the higher loadings, leading to incomplete evaporation during electrospinning. Subsequent evaporation of the residual solvent during storage leads to the formation of flattened fibers (Koski et al., 2004). Some precipitation of the drug was also noted during spinning with the higher concentration VAL-containing solutions (V4, AV3; data not shown). Regardless of this, no drug crystals can be seen on the fiber surfaces.

The diameters of the fibers are detailed in Table 2. When the drug loading percentage was increased, the fiber diameter rises. This is as expected, since an increase in drug content results in an increase in the amount of mass expelled from the spinneret per unit time, and is in good agreement with literature data on the electrospinning of PVP fibers loaded (Illangakoon et al., 2014; Lopez et al., 2014; Yu et al., 2009)

\subsection{Mat thickness}

The thickness of the fiber mats was measured for $4 \mathrm{~cm}$ circular cuts taken from different locations with each formulation (Table 2). The data show an increase in thickness for formulation $A 0$ to $A 4$ as the $A B$ loading rises from 0 to $55 \%$. In contrast, with $\mathrm{V} 0-\mathrm{V} 4$ the thickness of the mat decreases as the loading increases. In the combined-drug AV formulations the average mat thickness again increases with the drug content. These changes in thickness may be to the different shapes of the fibers in each formulation. The $A B$ and $A B / V A L$ fibers are generally all cylindrical, except for $A V 3$. In contrast, there is a move to flat ribbon-like fibers with an increasing drug loading in the VAL systems (see Figure 1). Inside the fiber mat, the cylindrical fibers occupy a greater vertical bulk compared to the ribbon-like fibers. When increasing the loading of $A B$ from $A 0$ to $A 4$ the fibers become thicker, causing the mat to thicken in turn. In contrast, increasing the VAL loading from V0 to V4 led to more flattened fibers accumulating and a thinner mat. A balance of these factors is operational in the AV systems, resulting overall in a small increase in thickness with the drug loading.

For oral fast dissolving formulations, the optimised film thickness range has been proposed to be between 20-500 $\mu \mathrm{m}$ (Bala et al., 2013). The results in Table 2 show that the mats prepared in this work fall within this range, and suggests there is the flexibility to increase the collection time to produce thicker mats with a larger mass of drug incorporated if required.

\subsection{Folding endurance}

Folding endurance is mechanical property used to characterize the ability of thin films to resist cracking or breaking upon packaging, storing and patient usage (Vuddanda et al., 2016). Folding endurance results are reported in Table 2. The folding endurance of the fibers decreases with a rising drug loading, as a result of the fibers becoming more brittle and mechanically weaker (Bölgen et al., 2005). However, all the values are generally high, and compare favourably with the literature (Illangakoon et al., 2014). 
This indicates that all the formulations prepared have appropriate mechanical properties to find application as oral films.

Table 2: Characterising data for the fiber mats. Diameters are calculated from 100 measurements in ImageJ. Thickness and folding endurance data are from three repeat experiments. All data are presented as mean \pm S.D.

\begin{tabular}{cccc}
\hline Formulation & Fiber diameter $(\mathbf{n m})$ & Thickness $(\boldsymbol{\mu m})$ & Folding endurance (number of folds) \\
\hline Blank PVP fibers & -- & $320 \pm 26$ & $61 \pm 2$ \\
\hline A1 & $344 \pm 70$ & $338 \pm 50$ & $54 \pm 15$ \\
A2 & $474 \pm 86$ & $340 \pm 30$ & $51 \pm 10$ \\
A3 & $880 \pm 294$ & $352 \pm 60$ & $35 \pm 4$ \\
A4 & $1230 \pm 400$ & $450 \pm 100$ & $30 \pm 4$ \\
\hline V1 & $724 \pm 120$ & $330 \pm 51$ & $80 \pm 1$ \\
V2 & $741 \pm 107$ & $206 \pm 20$ & $72 \pm 16$ \\
V3 & $882 \pm 127$ & $146 \pm 25$ & $46 \pm 11$ \\
V4 & $909 \pm 384$ & $150 \pm 10$ & $17 \pm 6$ \\
\hline AV1 & $461 \pm 72$ & $160 \pm 10$ & $80 \pm 34$ \\
AV2 & $737 \pm 154$ & $210 \pm 10$ & $36 \pm 4$ \\
AV3 & $1270 \pm 278$ & $260 \pm 20$ & $17 \pm 9$ \\
\hline
\end{tabular}

\subsection{Physical form}

The physical form of the drug components in the nanofibers was examined by XRD and DSC. XRD results are given in Figure 3. The $A B$ pattern displays a series of sharp Bragg reflections consistent with its anhydrous form (Koradia et al., 2010). VAL displays some reflections, but these are weak and broad. It is known that it is challenging to prepare highly crystalline VAL, and commercial materials show poor crystallinity (Wang et al., 2013). These observations are therefore all consistent with the literature. In contrast, PVP is clearly amorphous with only broad haloes in its diffraction pattern.

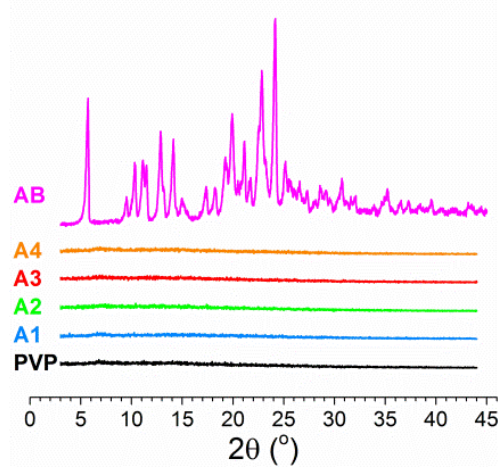

(a)

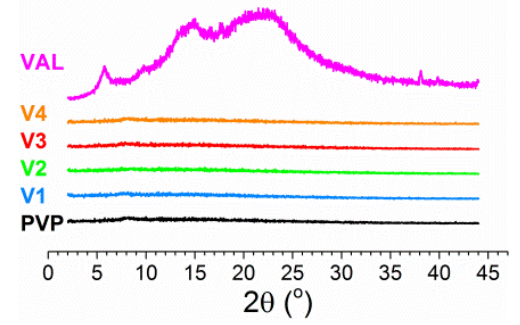

(b)

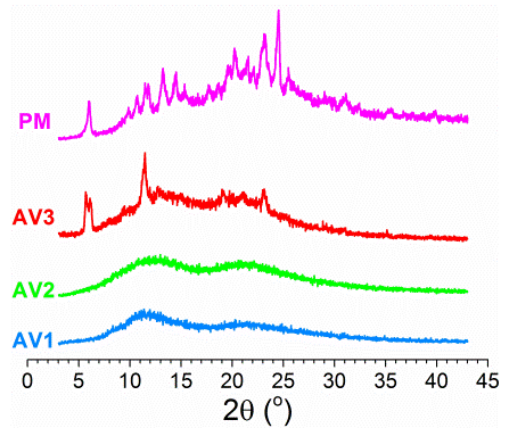

(c)

Figure 2: XRD diffraction patterns of the nanofiber formulations and raw materials. (a) AB-loaded fibers; (b) VAL-loaded fibers; and (c) AB/VAL dual drug systems. PM: physical mixture (30\% AB / 30\% VAL / 40\% PVP by mass).

All the electrospun fibers loaded with AB (Figure 2(a)) and VAL (Figure 2(b)) show no Bragg reflections, demonstrating that the fibers comprise amorphous solid dispersions. In the patterns of AV1 and AV2, the characteristic reflections of $A B$ and VAL have again disappeared, consistent with the formation of an amorphous system (Figure 2(c)). The formation of amorphous solid dispersions after electrospinning has 


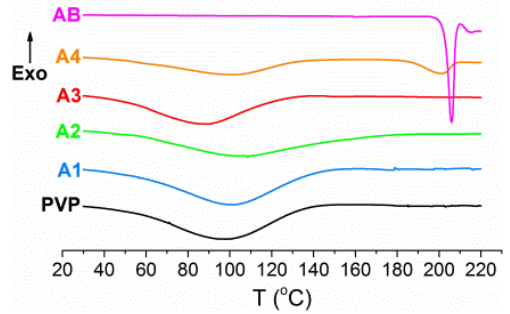

(a)

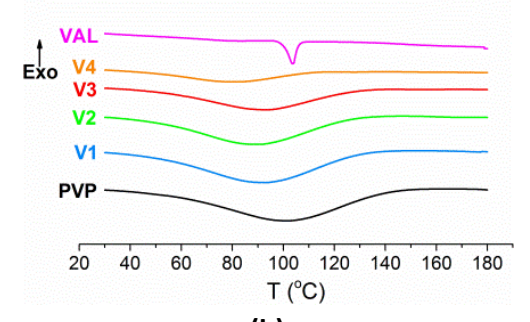

(b)

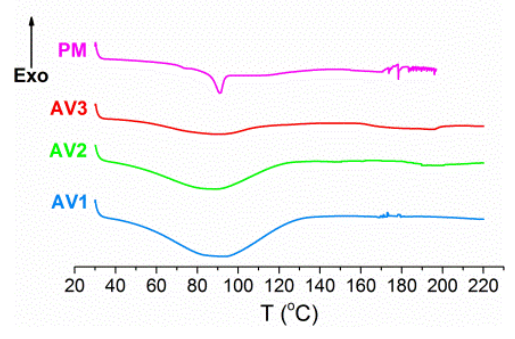

(c) 


\subsection{Infrared spectroscopy}

IR spectra were collected to investigate the drug-polymer interactions and help understand the compatibility of the APIs with the polymer in the fibers. Compatibility is essential for producing high quality and stable nanocomposites, because solid phase separation might occur if the API is not compatible with the polymer. The molecular structures of $A B$ and VAL are shown in Figure 4(a). Fulllength IR spectra can be found in Figure S2, while enlargements of the carboxylate region are given in Figure 4(b)-(d).

The IR spectrum of $A B$ shows characteristic $C=0$ stretches at $1697 \mathrm{~cm}^{-1}$ and $1673 \mathrm{~cm}^{-1}$, and a sharp peak at $1089 \mathrm{~cm}^{-1}$ with a shoulder at $1114 \mathrm{~cm}^{-1}$ from the C-O-C asymmetric stretch. Other peaks are present at $1048 \mathrm{~cm}^{-1}$ ( $\mathrm{NH}_{3}$ wagging), at $869 \mathrm{~cm}^{-1}$ (-C-O in plane bending), and at $753 \mathrm{~cm}^{-1}, 728 \mathrm{~cm}^{-1}$, and $689 \mathrm{~cm}^{-1}$ for ( $\mathrm{N}-\mathrm{H}$ vibrations). The IR spectrum of VAL has characteristic peaks at $3300 \mathrm{~cm}^{-1}(\mathrm{O}-\mathrm{H}$, and $\mathrm{N}-\mathrm{H}$ stretching vibrations), and at $2963 \mathrm{~cm}^{-1}$ (aromatic $\mathrm{CH}_{2}$ stretching vibration), $1730 \mathrm{~cm}^{-1}$ ( $\mathrm{C}=\mathrm{O}$ carbonyl vibration), $1603 \mathrm{~cm}^{-1}$ ( $\mathrm{N}-\mathrm{C}=\mathrm{O}$ amide carbonyl stretching), and 1451 and $1470 \mathrm{~cm}^{-1}$ (aromatic C=C vibrations). Pure PVP shows bands from $3650-3050 \mathrm{~cm}^{1}$ (O-H stretches from adsorbed water), 2840 $3010 \mathrm{~cm}^{1}$ (C-H stretches), $1660 \mathrm{~cm}^{1}$ (C=O) and $1290 \mathrm{~cm}^{1}$ (C-N stretch).

The spectra of the electrospun fibers show significant changes from the raw materials. These are most noticeable in the spectra of the highest drug loading formulations. The $A B C=O$ stretches at $1697 \mathrm{~cm}^{-1}$ and $1673 \mathrm{~cm}^{-1}$ have both merged into the PVP C=O peak at $1660 \mathrm{~cm}^{-1}$. Similarly, the $\mathrm{C}-\mathrm{O}-\mathrm{C}$ vibration at $1114 \mathrm{~cm}^{-1}$ in $A B$ shifts to $1123 \mathrm{~cm}^{-1}$ in the fibers. In the spectrum of V4, the VAL peaks present at 1603 $\mathrm{cm}^{-1}$ ( $\mathrm{N}-\mathrm{C}=\mathrm{O}$ amide carbonyl stretching) and $1470 \mathrm{~cm}^{-1}$ (aromatic $\mathrm{C}=\mathrm{C}$ ) for the pure drug are shifted to $1637 \mathrm{~cm}^{-1}$ and $1461 \mathrm{~cm}^{-1}$. Similar findings are noted for the FDC fibers. 


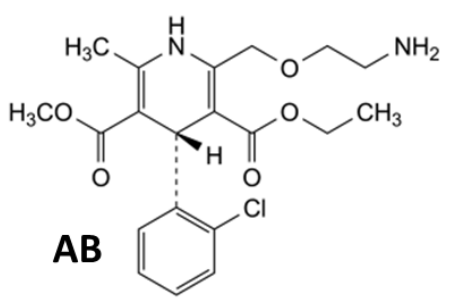

(

(a)

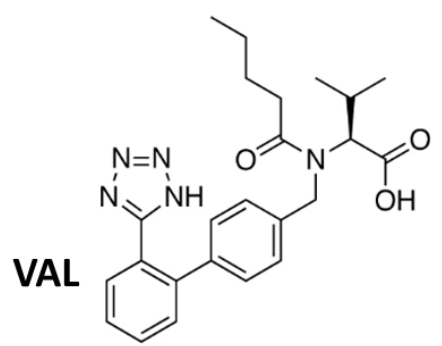

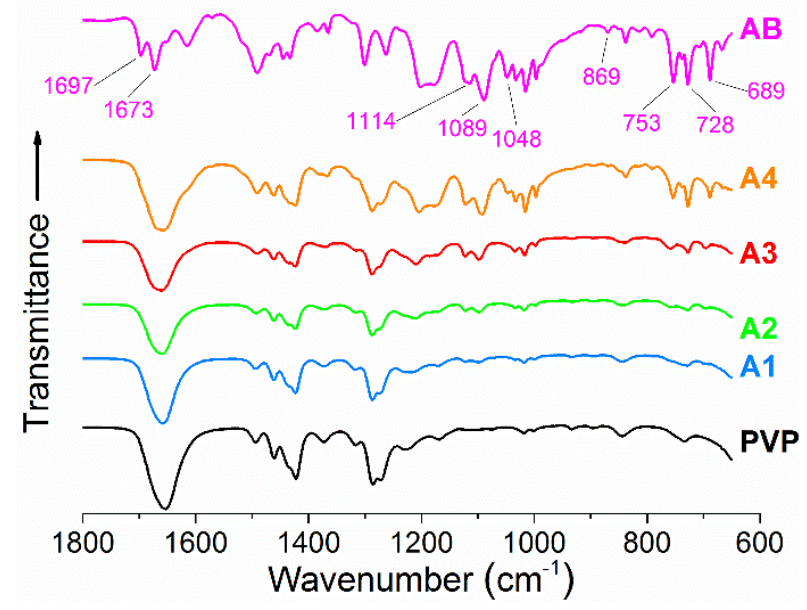

(b)
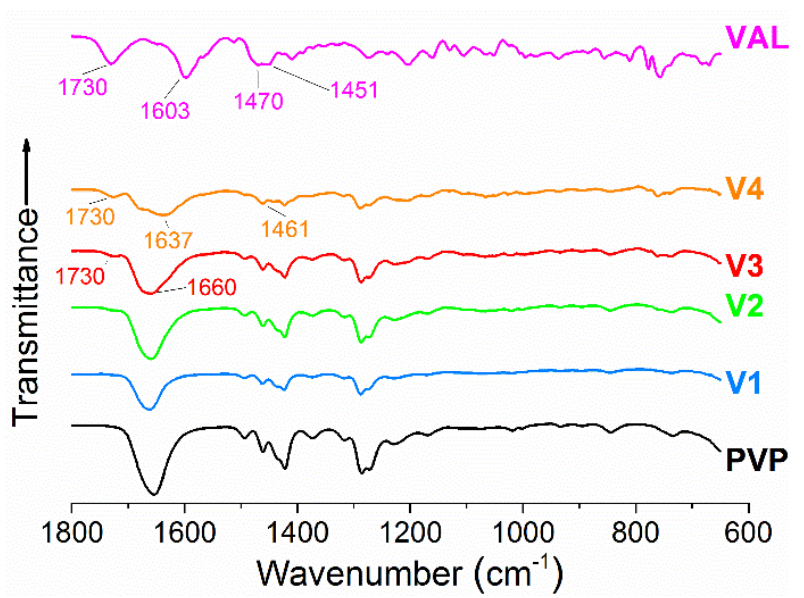

(c)

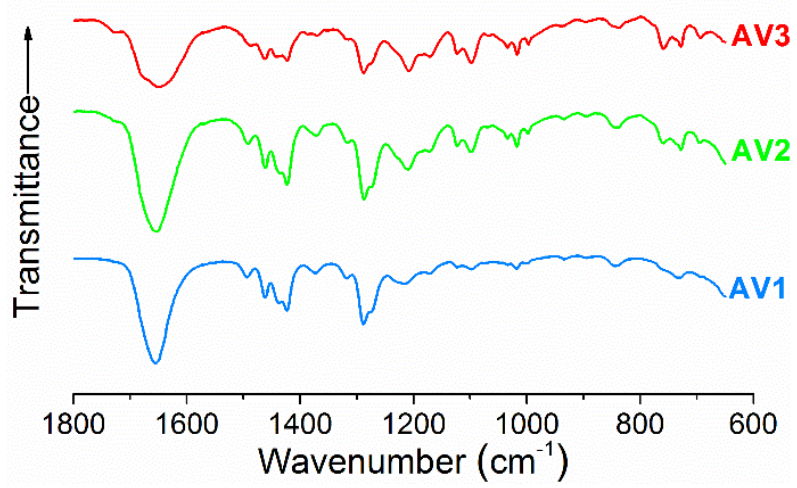

(d)

Figure 4. (a) The chemical structures of $A B$ and VAL, together with IR spectra for the (b) $A B$; (c) VAL; and, (d) FDC fibers. PM: $A$ physical mixture of $A B, V A L$, and PVP.

All these observations indicate the formation of intermolecular bonds between the drugs and PVP (Chen et al., 2008). The formation of interactions such as hydrogen bonding (between e.g. $\mathrm{N}-\mathrm{H}$ and O-H groups in $A B$ and $V A L$ and the $C=O$ groups of $P V P$ ), van der Waals interactions, and other secondary interactions such hydrophobic interactions (between the aromatic groups of the drugs and polymer), should lead to good compatibility between the components in the fiber mat (Wu et al., 2015) and aid the long term stability of the formulations (Mukherjee et al., 2005). 


\subsection{Drug loading and entrapment efficiency}

354 The drug loading and encapsulation efficiencies were determined by HPLC (see Table 4). All the 355 entrapment efficiencies are $\geq 85 \%$, with most very close to $100 \%$. This is as expected, since there is 356 minimal scope for loss of material in the electrospinning experiment unless there is precipitation in the 357 syringe. In some cases, it can be seen that the EE values are $>100 \%$; we ascribe this to a degree of 358 batch-to-batch variability in the formulations, particularly in terms of the amount of water present (PVP 359 is hygroscopic, and so will absorb water).

360

361

362

Table 4. The drug loadings and encapsulation efficiencies (EE) of the fibers. Data are reported as mean \pm S.D. from three experiments.

\begin{tabular}{ccccc}
\hline Formulation & AB loading $(\% \mathbf{w} / \mathbf{w})$ & AB EE $(\% \mathbf{w} / \mathbf{w})$ & VAL loading $(\% \mathbf{w} / \mathbf{w})$ & VAL EE (\%) \\
\hline A1 & $5.3 \pm 0.3$ & $107.3 \pm 5.8$ & - & - \\
A2 & $14.4 \pm 0.8$ & $96.2 \pm 5.4$ & - & - \\
A3 & $26.0 \pm 3.4$ & $86.8 \pm 11.2$ & - & - \\
A4 & $56.6 \pm 1.1$ & $102.9 \pm 2.1$ & - & - \\
\hline V1 & - & - & $4.5 \pm 0.2$ & $90.0 \pm 0.5$ \\
V2 & - & - & $14.8 \pm 0.6$ & $98.6 \pm 3.9$ \\
V3 & - & - & $29.7 \pm 0.3$ & $99.0 \pm 0.9$ \\
V4 & - & - & $54.7 \pm 2.3$ & $99.5 \pm 4.2$ \\
\hline AV1 & $5.4 \pm 0.3$ & $108.9 \pm 5.0$ & $5.48 \pm 0.2$ & $109.7 \pm 4.0$ \\
AV2 & $14.6 \pm 0.3$ & $97.1 \pm 2.3$ & $14.99 \pm 0.5$ & $99.9 \pm 3.4$ \\
AV3 & $27.0 \pm 0.4$ & $90.0 \pm 1.4$ & $31.32 \pm 0.7$ & $104.4 \pm 2.4$ \\
\hline
\end{tabular}

363

364

365

366

367

368

369

370

371

372

\subsection{Wetting assays}

In this study a minimal volume of SS was used to simulate the amount of moisture in the mouth and the time taken for disintegration determined for $A 1$ and $A 3$ using a high speed camera (Figure 5). Complete disintegration is observed in $<1 \mathrm{~s}$ for $\mathrm{A} 1$, and $<3 \mathrm{~s}$ for $\mathrm{A} 3$. This is slightly slower than previously reported for PVP-based fibers (Illangakoon et al., 2014), but nevertheless very promising for fast dissolving films. Results from other studies on oral fast dissolving formulations reported a range of disintegration times between 8 and 20 seconds (Cilurzo et al., 2011; Yu et al., 2009). 


\section{Formulation A1}

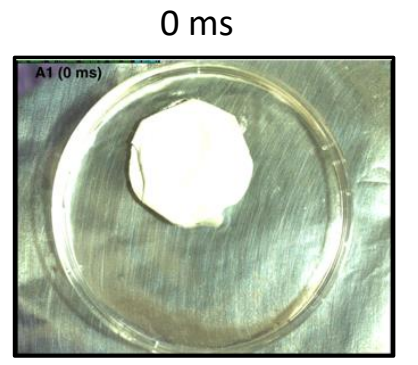

$600 \mathrm{~ms}$

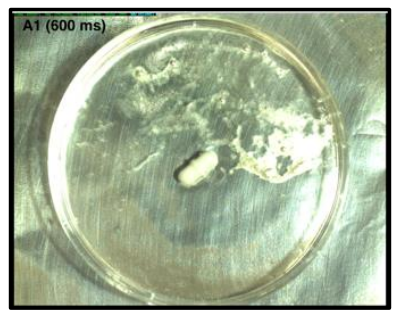

$0 \mathrm{~ms}$

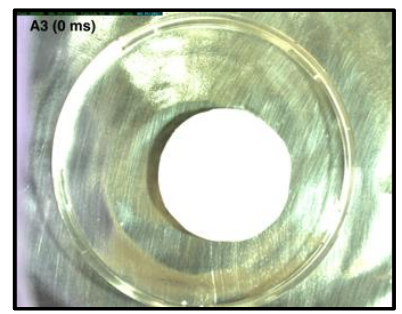

$600 \mathrm{~ms}$

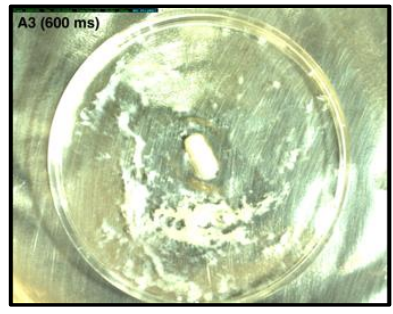

$100 \mathrm{~ms}$

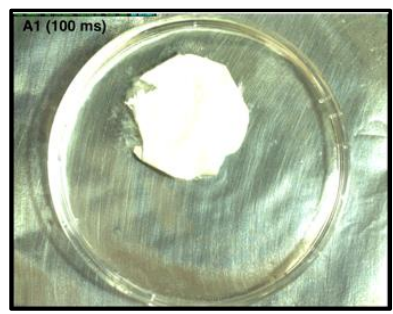

$800 \mathrm{~ms}$

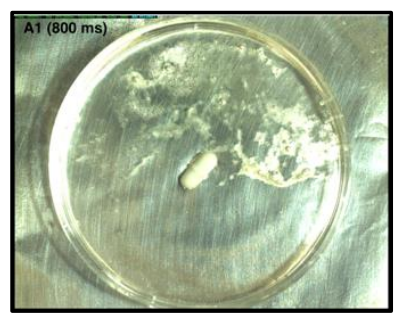

$200 \mathrm{~ms}$

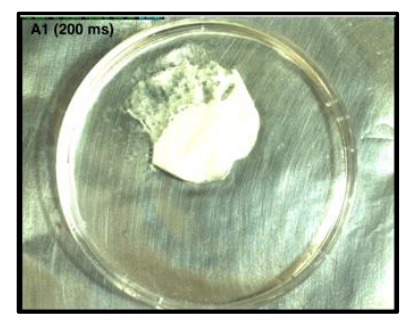

$100 \mathrm{~ms}$

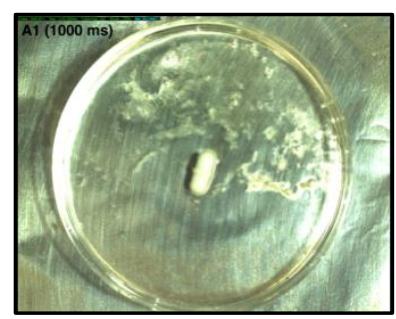

Formulation A3

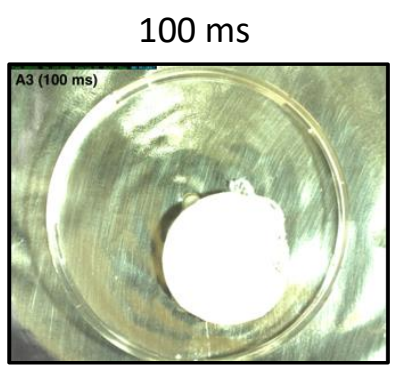

$800 \mathrm{~ms}$

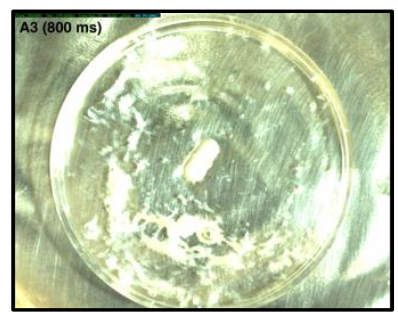

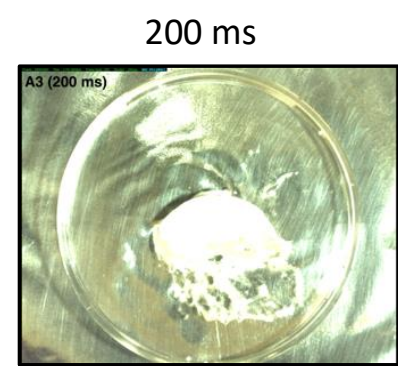

$1000 \mathrm{~ms}$

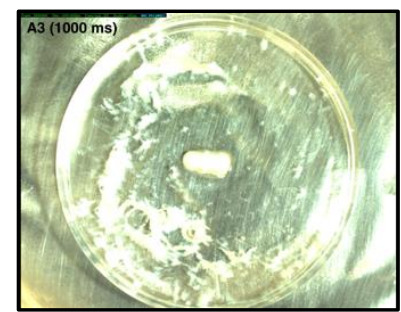

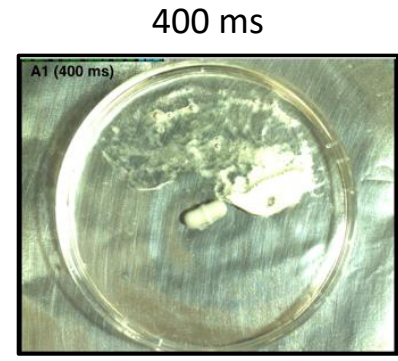

$3000 \mathrm{~ms}$

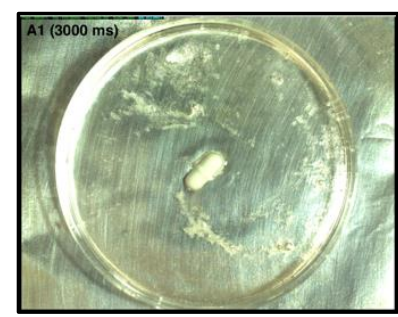

$400 \mathrm{~ms}$

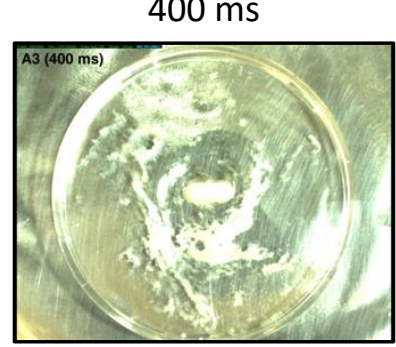

$3000 \mathrm{~ms}$

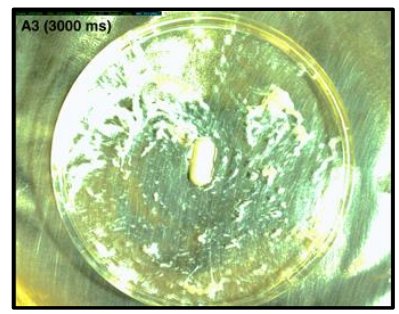

Figure 5: High speed camera images of the disintegration of $A 1$ and $A 3$ in simulated saliva.

\subsection{In vitro drug release}

In vitro dissolution tests were carried out in artificial simulated saliva. The results are given in Figure 6 . In some cases release percentages a little over $100 \%$ are observed; this can be attributed to a degree of batch-to-batch variability in the formulations. Release of $A B$ from all its single-drug fibers was very rapid: over $90 \%$ of the loading was released after $120 \mathrm{~s}$ for $\mathrm{A} 1, \mathrm{~A} 2$ and $\mathrm{A} 3$, and after $270 \mathrm{~s}$ with $\mathrm{A} 4$ (see Figure 6(a)). Similar findings were obtained in a study by (Yu et al., 2009) on PVP electrospun fibers loaded) with ibuprofen, where an increase in drug loading also extended the release time. 


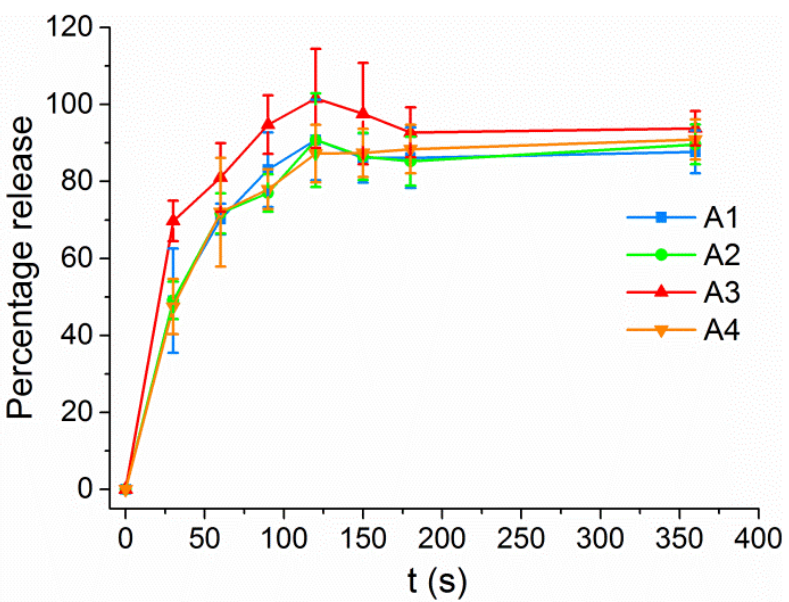

(a)

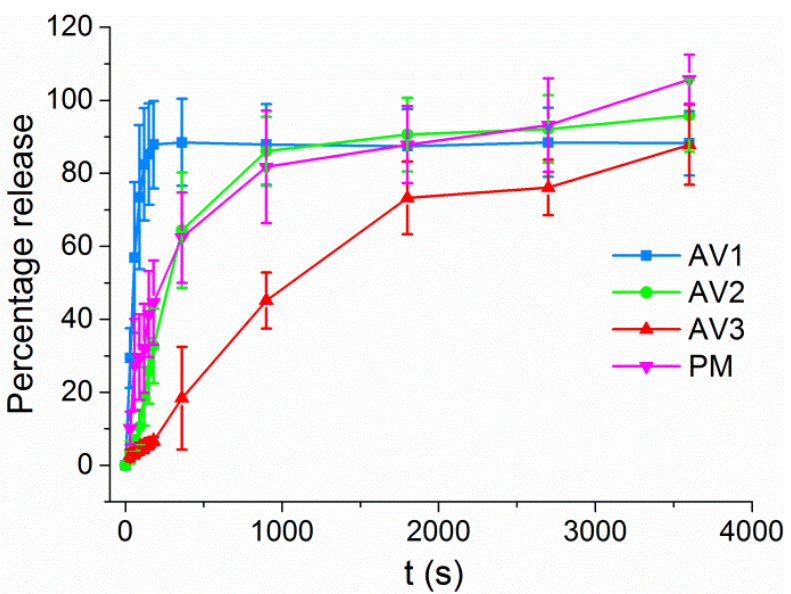

(c)

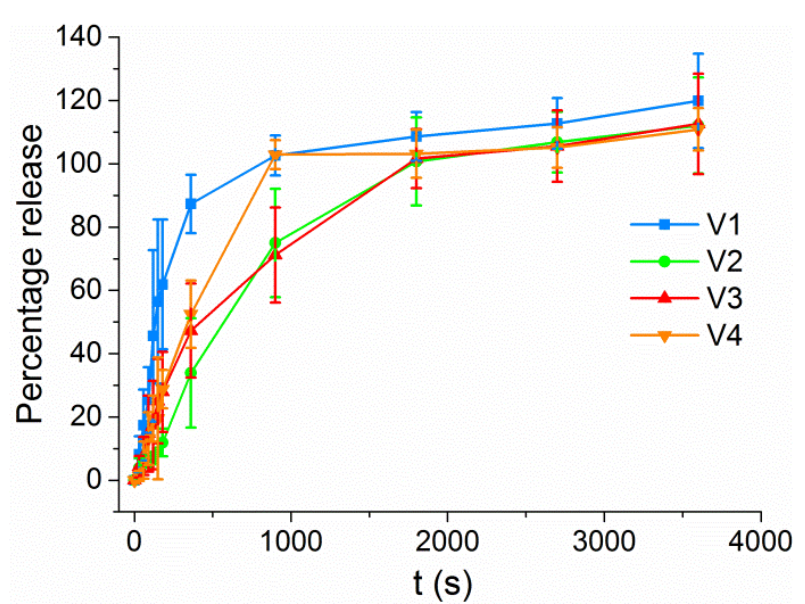

(b)

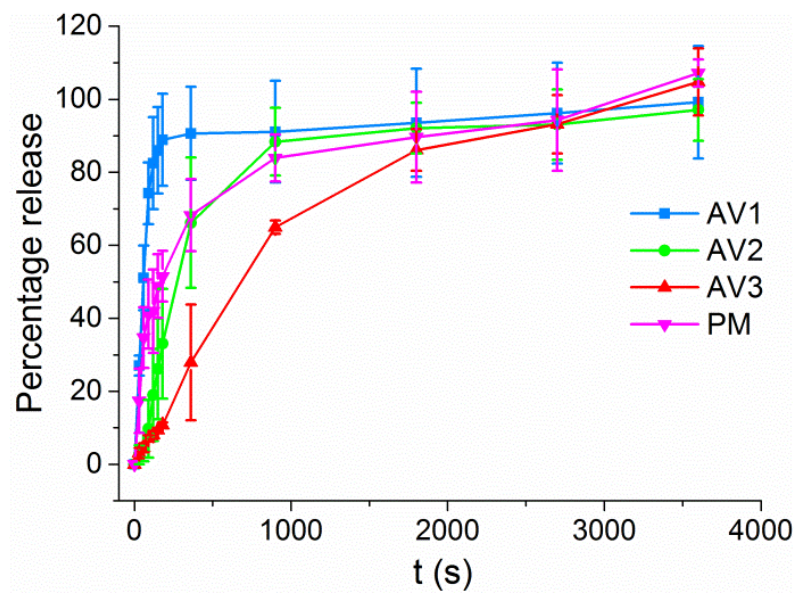

(d)
384

385

386

387

388

389

390

391

392

393

394

395

396

397

398

399

400

401

Figure 6: In vitro dissolution profiles showing (a) release of $A B$ from $A 1$ to $A 4$; (b) release of VAL from V1 to V4; (c) release of $A B$ from the FDC fiber mats; and, (d) release of VAL from FDC fibers. PM: physical mixture ( $30 \% A B / 30 \%$ VAL / 40\% PVP by mass).

The release of VAL from V1 - V4 was much slower, however (Figure 6(b)). Drug release from V1 reached $90 \%$ only after $370 \mathrm{~s}$, slightly over $6 \mathrm{~min}$. The other materials were even slower to release, with V4 not attaining $90 \%$ release until some $24 \mathrm{~min}$ after the start of the experiment. The key factor controlling release from V1 is the presence of 95\% w/w PVP in the fibers, which enhances the disintegration of the fibers and the dissolving of VAL into solution. As the VAL loading increases, the PVP content in the fibers declines, and thus disintegration and dissolution slow down. A similar lengthening of the release time is noted with $A B$, but this is much less significant because $A B$ is a salt and thus has substantially higher solubility than VAL.

In the combined-drug AV formulations, from each FDC the release profiles of both APIs (see Figure 6(c) and (d)) are very similar. With AV1, fast release of both drugs is seen, and $360 \mathrm{~s}$ into the experiment release reaches $88 \pm 12 \%$ for $A B$, and $90 \pm 13 \%$ for VAL. This presumably arises because of the high content of the hydrophilic polymer PVP and the molecular dispersion of $A B$ and VAL in it. In addition to VAL AV1 contains PVP and also highly soluble AB; the presence of these two hydrophilic entities (cf just PVP and VAL in V1) accelerated the disintegration of the formulation and freeing of VAL into solution. 


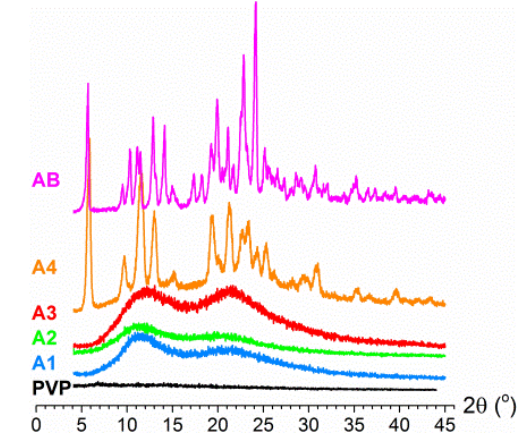

(a)

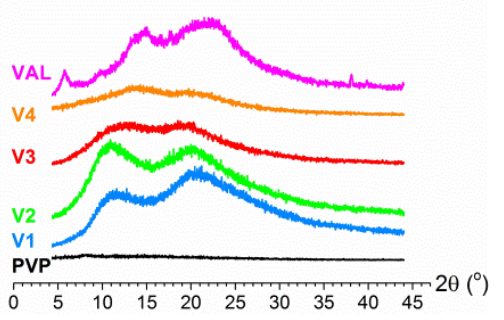

(b)

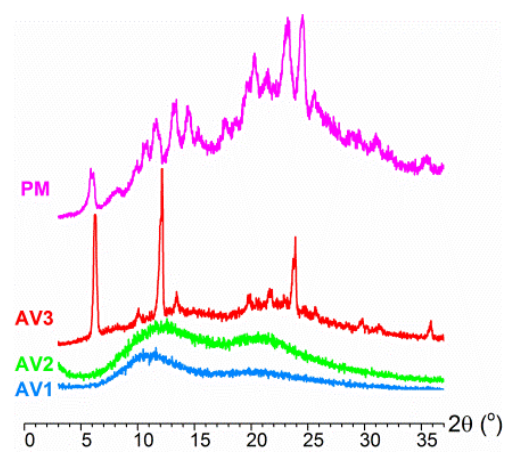

(c)

Formulation AV1 is potentially therefore a good choice for fabricating fast dissolving FDC formulation As for the single-drug fibers, an increasing drug loading leads to a slowing in the rate of drug dissolution, however. In AV2, 90\% release of AB was attained only after $1800 \mathrm{~s}$ (30 min), and 90\% of the VAL content after $900 \mathrm{~s}$. For $A V 3$, the $90 \%$ release times $>3600 \mathrm{~s}$ for $A B$ and $2190 \mathrm{~s}$ for VAL. All these times are much too long for an effective oral film, and cannot be said to be fast dissolving. Indeed, AV3 dissolves more slowly than an analogous physical mixture of the drugs and PVP (see Figure 7(c) and (d)). The reasons behind this are not completely clear, but it is evident from the XRD data (Figure 2(c)) that AV3 contains crystalline material. This, coupled with drug/polymer interactions and a relatively slow disentanglement of the PVP polymer molecules when the formulation is added to water, is expected to be responsible for slowing the rate of drug release over the physical mixture.

\subsection{Stability study}

Since amorphous materials will seek to relax to a crystalline state over time, the storage stability of the fibers was explored. XRD data were recorded on fibers which had been stored in a desiccator for 4 months (see Figure 7). The diffraction data clearly show the bulk of the formulations remain amorphous over this time, and distinct Bragg reflections can only be seen for A4 and AV3. In both of these cases there is very clear evidence for the recrystallization of $A B$. VAL recrystallization cannot be ascertained, but this is not surprising since VAL is known to be poorly crystalline, and also the presence of ions in $A B$ will encourage more rapid reversion to an ordered form. It appears that phase separation may be starting to occur after 4 months' ageing for A4 and AV3, a phenomenon also noticed by Lopez et al.,

Figure 7: Physical characterisation data obtained on aged materials. XRD data are shown for the (a) AB; (b) VAL; and, (c) FDC fibers.

Overall, it is clear from this study that electrospun nanofibers can be loaded with multiple drugs to provide rapid release in conditions representative of the mouth, and that at lower doses the fibers are stable upon storage for at least four months. They thus offer a smart drug delivery platform for treating co-morbidities in the elderly population. The drug release profile was found to be dependent on the drug loading, and these results will be helpful to guide future studies aiming to fabricate immediate release FDC formulations for co-morbidities. The fast dissolving feature of the electrospun fiber systems 
reported here is also expected to lead to high acceptability by patients with swallowing difficulties, and hence to lead to higher adherence.

\section{Conclusions}

The aim of this study was to utilize electrospinning to prepare FDC fast-dissolving drug delivery systems. We were able to develop nanofibers with drug loadings between $5 \%$ and $55 \% \mathrm{w} / \mathrm{w}$. Two types of formulation were made, loaded either with a single drug (valsartan or amlodipine besylate), or both. Characterisation the fibers by scanning electron microscopy showed most to be cylindrical with smooth surfaces, but with a tendency to flatten with increased valsartan loadings. The fiber mats obtained had thicknesses between 146 and $450 \mu \mathrm{m}$, and most showed acceptable folding endurance except at very high drug loadings. $R$ studies revealed intermolecular interactions between $A B, V A L$, and the polymer PVP in the composite material, and X-ray diffraction and differential scanning calorimetry showed the majority of the formulations to comprise amorphous solid dispersions. Entrapment efficiencies were greater than $85 \%$ in all cases. The fiber mats wet and disintegrate in under $3 \mathrm{~s}$, indicating they have promise as oral fast-dissolving films. In vitro dissolution studies demonstrated that the amlodipine besylate-loaded fibers had fast release profiles freeing $90 \%$ of their drug cargo within $120 \mathrm{~s}$ with loadings of $30 \% \mathrm{w} / \mathrm{w}$ and below, or $360 \mathrm{~s}$ from s 55\% w/w formulation. In contrast, the valsartan formulations released their drug cargo much more slowly, with even $5 \% \mathrm{w} / \mathrm{w}$ fibers only reaching $90 \%$ release at $c a .360 \mathrm{~s}$, while the $55 \% \mathrm{w} / \mathrm{w}$ fiber required $24 \mathrm{~min}$ to reach this point. In the fixed dose combination fibers, the drug release profile varied markedly with the drug loading. $90 \%$ release of both drugs was reached within $360 \mathrm{~s}$ was achieved when the loading of each was $5 \% \mathrm{w} / \mathrm{w}$, but the rate declines rapidly as more drug is added. Except for those with the highest drug loadings, the fibers remain amorphous for at least 4 months' storage in a dessicator.

\section{Acknowledgements}

The authors thank the Umm AlQura University (Kingdom of Saudi Arabia) for provision of a scholarship to HB. We also gratefully acknowledge Mr Dave McCarthy and Mrs Kate Keen for assistance with obtaining SEM images, and Dr Asma Buanz for guidance on DSC experiments.

\section{References}

Adeli, E., 2015. Irbesartan-loaded electrospun nanofibers-based PVP K90 for the drug dissolution improvement: Fabrication, in vitro performance assessment, and in vivo evaluation. J. Appl. Polym. Sci. 132, 42212.

Bala, R., Pawar, P., Khanna, S., Arora, S., 2013. Orally dissolving strips: A new approach to oral drug delivery system. Int. J. Pharm. Investig. 3, 67-76.

Bangalore, S., Kamalakkannan, G., Parkar, S., Messerli, F.H., 2007. Fixed-dose combinations improve medication compliance: a meta-analysis. Am. J. Med. 120, 713-719.

Bölgen, N., Menceloğlu, Y.Z., Acatay, K., Vargel, i., Pişkin, E., 2005. In vitro and in vivo degradation of non-woven materials made of poly ( $\varepsilon$-caprolactone) nanofibers prepared by electrospinning under different conditions. J. Biomed. Sci. Polym. Ed. 16, 1537-1555. 
Celebier, M., Kaynak, M.S., Altınöz, S., Sahin, S., 2010. HPLC method development for the simultaneous analysis of amlodipine and valsartan in combined dosage forms and in vitro dissolution studies. Brazil. J. Pharm. Sci 46, 761-768.

Chen, J., Wang, X., Zhang, W., Yu, S., Fan, J., Cheng, B., Yang, X., Pan, W., 2016. A novel application of electrospinning technique in sublingual membrane: characterization, permeation and in vivo study. Drug. Dev. Ind. Pharm. 42, 1365-1374.

Chen, Z., Mo, X., He, C., Wang, H., 2008. Intermolecular interactions in electrospun collagen-chitosan complex nanofibers. Carbohydr. Polym. 72, 410-418.

Cilurzo, F., Cupone, I.E., Minghetti, P., Buratti, S., Gennari, C.G., Montanari, L., 2011. Diclofenac fastdissolving film: suppression of bitterness by a taste-sensing system. Drug Dev. Ind. Pharm. 37, 252-259. Desai, D., Wang, J., Wen, H., Li, X., Timmins, P., 2013. Formulation design, challenges, and development considerations for fixed dose combination (FDC) of oral solid dosage forms. Pharm. Dev. Technol. 18, 1265-1276.

European Medicines Agency, 2017. Reflection paper on the pharmaceutical development of medicines for use in the older population.

Fulton, M.M., Allen, E., 2005. Polypharmacy in the elderly: a literature review. J. Am. Acad. Nurse Pract. 17, 123-132.

Gorard, D.A., 2006. Escalating polypharmacy. QJM 99, 797-800.

http://www.chemicalbook.com/ChemicalProductProperty US CB4127875.aspx.

https://pubchem.ncbi.nlm.nih.gov/compound/valsartan\#section=Top.

Hughes, C.M., 2004. Medication non-adherence in the elderly. Drugs \& Aging 21, 793-811.

Illangakoon, U.E., Gill, H., Shearman, G.C., Parhizkar, M., Mahalingam, S., Chatterton, N.P., Williams, G.R., 2014. Fast dissolving paracetamol/caffeine nanofibers prepared by electrospinning. Int. J. Pharm. 477, 369-379.

Jimmy, B., Jose, J., 2011. Patient medication adherence: measures in daily practice. Oman Med. J. 26, 155-159.

Jin, M., Yu, D.G., Geraldes, C.F., Williams, G.R., Bligh, S.W., 2016. Theranostic fibers for simultaneous imaging and drug delivery. Mol. Pharm. 13, 2457-2465.

Khaled, S.A., Burley, J.C., Alexander, M.R., Yang, J., Roberts, C.J., 2015. 3D printing of five-in-one dose combination polypill with defined immediate and sustained release profiles. J. Controlled Release 217, 308-314.

Koradia, V., Lopez de Diego, H., Frydenvang, K., Ringkjøbing-Elema, K., Müllertz, A., Bond, A.D., Rantanen, J., 2010. Solid forms of amlodipine besylate: Physicochemical, structural, and thermodynamic characterization. Cryst. Growth Des. 10, 5279-5290.

Koski, A., Yim, K., Shivkumar, S., 2004. Effect of molecular weight on fibrous PVA produced by electrospinning. Mater. Lett. 58, 493-497.

Li, X., Kanjwal, M.A., Lin, L., Chronakis, I.S., 2013a. Electrospun polyvinyl-alcohol nanofibers as oral fastdissolving delivery system of caffeine and riboflavin. Colloids Surf. B 103, 182-188.

Li, X., Lin, L., Zhu, Y., Liu, W., Yu, T., Ge, M., 2013b. Preparation of ultrafine fast-dissolving cholecalciferol-loaded poly(vinyl pyrrolidone) fiber mats via electrospinning. Polym. Composite. 34, 282287.

Liu, F., Ranmal, S., Batchelor, H.K., Orlu-Gul, M., Ernest, T.B., Thomas, I.W., Flanagan, T., Tuleu, C., 2014. Patient-centred pharmaceutical design to improve acceptability of medicines: similarities and differences in paediatric and geriatric populations. Drugs 74, 1871-1889. 
Lopez, F.L., Shearman, G.C., Gaisford, S., Williams, G.R., 2014. Amorphous formulations of indomethacin and griseofulvin prepared by electrospinning. Mol. Pharm. 11, 4327-4338.

Mitra, A., Wu, Y., 2012. Challenges and opportunities in achieving bioequivalence for fixed-dose combination products. AAPS J. 14, 646-655.

Mukherjee, B., Mahapatra, S., Gupta, R., Patra, B., Tiwari, A., Arora, P., 2005. A comparison between povidone-ethylcellulose and povidone-eudragit transdermal dexamethasone matrix patches based on in vitro skin permeation. Eur. J. Pharm. Biopharm. 59, 475-483.

Mundargi, R.C., Patil, S.A., Agnihotri, S.A., Aminabhavi, T.M., 2007. Evaluation and controlled release characteristics of modified xanthan films for transdermal delivery of atenolol. Drug Dev. Ind. Pharm. 33, 79-90.

Nagy, Z.K., Nyul, K., Wagner, I., Molnar, K., Marosi, G., 2010. Electrospun water soluble polymer mat for ultrafast release of Donepezil HCl. Express Polym. Lett. 4, 763-772.

Plosker, G.L., Robinson, D.M., 2008. Amlodipine/Valsartan: fixed-dose combination in hypertension. Drugs 68, 373-381.

Seager, H., 1998. Drug-delivery products and the Zydis fast-dissolving dosage form. J. Pharm. Pharmacol. 50, 375-382.

Silva, A.C.M., Gálico, D.A., Guerra, R.B., Perpétuo, G.L., Legendre, A.O., Rinaldo, D., Bannach, G., 2014. Thermal stability and thermal decomposition of the antihypertensive drug amlodipine besylate. J. Therm. Anal. Calorim. 120, 889-892.

Vigh, T., Horvathova, T., Balogh, A., Soti, P.L., Dravavolgyi, G., Nagy, Z.K., Marosi, G., 2013. Polymer-free and polyvinylpirrolidone-based electrospun solid dosage forms for drug dissolution enhancement. Eur. J. Pharm. Sci. 49, 595-602.

Vuddanda, P.R., Mathew, A.P., Velaga, S., 2016. Electrospun nanofiber mats for ultrafast release of ondansetron. React. Funct. Polym. 99, 65-72.

Wang, J.-R., Wang, X., Lu, L., Mei, X., 2013. Highly crystalline forms of valsartan with superior physicochemical stability. Cryst. Growth Des. 13, 3261-3269.

Williams, G.R., Chatterton, N.P., Nazir, T., Yu, D.-G., Zhu, L.-M., Branford-White, C.J., 2012. Electrospun nanofibers in drug delivery: recent developments and perspectives. Therap. Deliv. 3, 515-533.

Wu, Y.-H., Yu, D.-G., Li, X.-Y., Diao, A.-H., Illangakoon, U.E., Williams, G.R., 2015. Fast-dissolving sweet sedative nanofiber membranes. J. Mater. Sci. 50, 3604-3613.

Yu, D.-G., Branford-White, C., Shen, X.-X., Zhang, X.-F., Zhu, L.-M., 2010. Solid dispersions of ketoprofen in drug-loaded electrospun nanofibers. J. Dispersion Sci. Technol. 31, 902-908.

Yu, D.G., Shen, X.X., Branford-White, C., White, K., Zhu, L.M., Bligh, S.W., 2009. Oral fast-dissolving drug delivery membranes prepared from electrospun polyvinylpyrrolidone ultrafine fibers. Nanotechnol. 20, 055104.

Zamani, M., Prabhakaran, M.P., Ramakrishna, S., 2013. Advances in drug delivery via electrospun and electrosprayed nanomaterials. Int. J. Nanomedicine 8, 2997-3017. 